Bundesgesundheitsbl 2020 - 63:322-331 https://doi.org/10.1007/s00103-020-03102-1 Online publiziert: 20. Februar 2020

(c) Der/die Autor(en) 2020

Stefan Karrasch ${ }^{1,2,3} \cdot$ Annette Peters ${ }^{1} \cdot$ Christa Meisinger $^{4,5} \cdot$ Maike Ferland $^{1} \cdot$ Lina Jaeschke ${ }^{6}$. Tobias Pischon ${ }^{6,7,8,9} \cdot$ Julia Fricke ${ }^{10} \cdot$ Thomas Keil $^{10,11,12}$. Sylvia Gastell ${ }^{13}$ - Matthias Schulze ${ }^{14} \cdot$ Mirko Brandes $^{15} \cdot$ Kathrin Günther $^{15}$. Oliver Kuß ${ }^{16}$. Tamara Schikowski ${ }^{17}$. Carina Emmel ${ }^{18} \cdot$ Karl-Heinz Jöckel $^{18}$. Karin B. Michels ${ }^{19}$. Claus-Werner Franzke ${ }^{19}$. Susan Langer ${ }^{20} \cdot$ Rafael Mikolajczyk $^{20}$. Annika Jagodzinski ${ }^{21,22} \cdot$ Heiko Becher ${ }^{23}$. Stefanie Castell ${ }^{24} \cdot$ Yvonne Kemmling $^{24}$. Sabina Waniek ${ }^{25}$. Wolfgang Lieb ${ }^{25} \cdot$ Kerstin Wirkner ${ }^{26,27} \cdot$ Markus Loeffler $^{26,27}$. Karin Halina Greiser ${ }^{28}$. Rudolf Kaaks ${ }^{28,29}$. Nicole Legath ${ }^{30} \cdot$ Klaus Berger $^{30}$. Sabine Schipf ${ }^{31}$. Wolfgang Hoffmann ${ }^{32}$. Anja Sedlmeier ${ }^{33}$. Michael Leitzmann ${ }^{33}$. Hermann Brenner ${ }^{34} \cdot$ Bernd Holleczek $^{35} \cdot$ Holger Schulz $^{1,3}$

\title{
Lungenfunktion in der NAKO Gesundheitsstudie: Methoden und erste Ergebnisse
}

Umwelt-Interaktionen sowie diesbezüglichen Risikofaktoren, die von Lebensstilfaktoren bis zur Luftverschmutzung reichen.

In einer Reihe von Ländern innerhalb und außerhalb Europas greift eine landesweite Beschreibung der Atemwegsgesundheit, einschließlich Lungenfunktion und Häufigkeit von Lungenerkrankungen, auf umfangreiche, zum Teil noch laufende bevölkerungsbezogene Studien mit homogenen und standardisierten Erhebungen zurück [4-8]. Hierbei wurden auch innerhalb westeuropäischer Länder deutliche Unterschiede in der respiratorischen Mortalität, in der Häufigkeit von Atemwegserkrankungen und -beschwerden sowie bei den zugrunde liegenden Risikofaktoren, wie etwa Rauchverhalten, beobachtet $[9,10]$. Dies deutet darauf hin, dass eine Extrapolation der Ergebnisse selbst aus Nachbarländern innerhalb Europas nicht ohne Weiteres möglich ist, vielmehr Unterschiede in Geografie, Demografie, Berufsspektrum und Gesundheitswesen zu berücksichtigen sind. Nur Ergebnisse aus länderspezifischen Bevölkerungsstichproben ermöglichen daher zielgenaue Maßnahmen zur Verbesserung der Atemwegsgesundheit auf Basis adäquater und präziser Daten.

Als beste Quelle für die Situation in Deutschland können derzeit das deutsche und das europäische Lungenweiß- buch betrachtet werden, jedoch basieren die darin enthaltenen Daten zur respiratorischen Krankheitslast in erster Linie auf Interviews oder Ergebnissen aus ausgewählten geografischen Regionen [10, 11]. Eine Erhebung des respiratorischen Gesundheitszustandes auf der Grundlage bundesweit einheitlich und standardisiert durchgeführter Lungenfunktionsuntersuchungen lag in Deutschland bislang nicht vor. Dementsprechend stellten sowohl das deutsche als auch das europäische Lungenweißbuch einen substanziellen Mangel an genauen und umfassenden Daten im Hinblick auf die respiratorische Gesundheit in europäischen Ländern und in Deutschland fest und erklärten, eine standardisierte Datenerhebung sei vorrangig und dringend anzustreben [10, 11].

In Deutschland stehen große populationsbasierte Datensätze für die Allgemeinbevölkerung in Form der Studie zur Gesundheit Erwachsener in Deutschland (DEGS; [12]) und der Studie „Gesundheit in Deutschland Aktuell“ (GEDA) zur Verfügung [13], welche auch Lungenerkrankungen wie Asthma bronchiale und chronische Bronchitis abdecken. Allerdings sind die Informationen über den respiratorischen Status in Bezug aufSymptome, Raucheranamnese oder Behandlung einschließlich Medikation limitiert,

insbesondere fehlen Lungenfunktionsder Autoren befinden sich auf der letzten Artikelseite. 
messungen. Jene Untersuchungen hingegen, die auch Lungenfunktionstests einschließen, sind bislang auf bestimmte Studienregionen beschränkt [14-16]. Auf der anderen Seite gibt es mehrere landesweite Studien, die eine standardisierte und avancierte Untersuchung der Lungenfunktion beinhalten. Diese sind jedoch überwiegend auf klinische Kohorten ausgewählter Patientengruppen fokussiert, die an einer bestimmten Lungenerkrankung leiden und zum Teil ein begrenztes Altersspektrum aufweisen, so etwa Studien zu chronisch obstruktiver Lungenerkrankung (COPD; COSYCONET) oder Asthma bronchiale (ALLIANCE; [17-19]). Diese Kohorten sind von herausragendem Wert für die eingehende Untersuchung der betreffenden Lungenerkrankung, decken hingegen vom Ansatz her wichtige Fragen der öffentlichen Gesundheit nicht ab, speziell den respiratorischen Status in der Allgemeinbevölkerung. Diesem Mangel soll die Lungenfunktionsuntersuchung in der NAKO Gesundheitsstudie abhelfen.

Ziel der vorliegenden Arbeit ist es, das Vorgehen in der NAKO bei der standardisierten Erfassung des respiratorischen Status mittels Spirometrie und Bestimmung des exhalierten Stickstoffmonoxids („fractional exhaled nitric oxide“, FeNO) darzustellen und in einer vorläufigen, orientierenden Auswertung erste Ergebnisse aus der Datenbasis zur Halbzeit der Basiserhebung zu präsentieren, nicht zuletzt zu dem Zweck, Forschungsgruppen zur künftigen produktiven Verwendung der NAKO-Daten zu ermutigen.

\section{Material und Methoden}

Im Rahmen der NAKO wurden 200.000 Teilnehmende aus verschiedenen Regionen Deutschlands in 18 Studienzentren untersucht. Insgesamt umfassten die hoch standardisierten Erhebungen ein breites Spektrum von Krankheiten und gesundheitsbezogenen Fragen, darunter neben Atemwegsgesundheit auch Allergien sowie die wichtigsten Komorbiditäten von Atemwegserkrankungen, insbesondere Herz-KreislaufErkrankungen, Krebs und Diabetes. Hinsichtlich des Umfangs der durchgeführten Erhebungen und Messungen wurden verschiedene Ebenen der Untersuchungstiefe festgelegt. Insbesondere erfolgte die Aufteilung in das Level-1Programm, welches alle 200.000 Studienteilnehmenden umfasste, und das Level2-Programm mit einer Teilstichprobe von 40.000 Studienteilnehmenden, die zusätzliche Untersuchungsmodule durchliefen. Die diesbezügliche Zuordnung erfolgte bereits vor Einladung der Teilnehmenden, spätere Änderungen waren bei Bedarf möglich [20].

Im Hinblick auf die Atemwegsgesundheit bilden den Kern der Erhebungen vor allem eine Spirometrie, vorgesehen bei allen Teilnehmenden (Level 1), sowie die Messung des FeNO, vorgesehen bei der Teilstichprobe von 40.000 Teilnehmenden (Level 2). Die Spirometrie liefert Messgrößen des Lungenvolumens sowie einer möglichen Atemflusseinschränkung bzw. bronchialen Obstruktion. Der FeNO-Messwert dient als klinisch gut etablierter Marker für eine Th2getriebene Entzündung in den Atemwegen (typisch bei Erkrankungen des atopischen Formenkreises, wie etwa allergischem Asthma bronchiale oder allergischer Rhinitis).

Alle Lungenfunktionsuntersuchungen wurden nach den jeweiligen nationalen und internationalen Leitlinien durchgeführt [21-24]. Vor den Messungen wurde in einer Anamnese das Vorliegen von Kontraindikationen für die Spirometrie erfasst. Um vor dem Hintergrund diesbezüglich teils uneinheitlicher internationaler Empfehlungen [21, 24-26] potenzielle Risiken im vorliegenden Studiensetting, d.h. ohne medizinische Indikation zur Spirometrie, so weit als möglich zu minimieren, umfassten diese exzessiv erhöhten Blutdruck, koronare Herzerkrankung, Aorten- oder Hirnaneurysma, Hirnblutung, Pneumothorax, Bluthusten oder Tuberkulose im Jahr vor der Untersuchung, Operationen an Bauch, Brustkorb, Gehirn, Auge oder Atemwegen in den 4 Wochen vor der Untersuchung sowie eine Schwangerschaft jenseits der 16. Schwangerschaftswoche. Für die FeNO-Messung wurden keine gesonderten Kontraindikationen definiert. In Zweifelsfällen entschied der Studienarzt des jeweiligen Studienzentrums über die Durchführung der Lungen- funktionsuntersuchung bzw. wurde auf die Untersuchung verzichtet. Es erfolgte keine Anweisung zur Modifikation einer möglichen aktuellen respiratorischen Medikation. Darüber hinaus wurden Symptome, die auf eine aktuelle respiratorische Allergie hinweisen, sowie Atemwegsinfektionen innerhalb der letzten drei Wochen vor der Untersuchung erfragt.

Das Vorliegen von Atemwegserkrankungen oder -beeinträchtigungen sowie Risikofaktoren wurde zudem mithilfe von standardisierten Fragebögen und Interviews erfasst, darunter eine vom Arzt diagnostizierte chronische Bronchitis oder COPD, Asthma bronchiale, Heuschnupfen, Raucherstatus und -historie, Infektionen der oberen oder unteren Atemwege im vergangenen Jahr, Schnarchen und Schlafapnoe, berufsbedingte Exposition gegenüber Einflüssen, die die Verwendung einer Atemschutzmaske erfordern, sowie eine Reihe von Atemwegssymptomen, die auf Heuschnupfen, Asthma bronchiale oder chronische Bronchitis hindeuten.

Für die Spirometrie wurden Easyon-PC-Geräte mit ultraschallbasierter Messtechnik (ndd Medizintechnik AG, Schweiz) verwendet. Die Untersuchung wurde aufrecht sitzend mit Nasenklammer und unter beherzter Motivation durch die Untersuchenden durchgeführt, um eine leitliniengerechte Spirometrie innerhalb von 5 forcierten Exspirationsmanövern $\mathrm{zu}$ erzielen. Für jede Spirometrie wurden mehr als 50 Ergebnisparameter aus den aufgezeichneten Flussvolumenkurven automatisch erfasst und mittels eines optimierten Exportprotokolls an das Datenintegrationszentrum der NAKO übertragen. Darüber hinaus wurden für jedes durchgeführte spirometrische Manöver jeweils die vollständigen Rohdaten der Volumenund Atemflusszeitkurven aufgezeichnet und an das Datenintegrationszentrum übertragen.

Für die FeNO-Messung wurden NIOX-Vero-Geräte auf Basis elektrochemischer Messtechnik verwendet (Circassia AG, Schweden). Die Messung erfolgte aufrecht sitzend ohne Nasenklammer unter Anweisung durch die Untersuchenden, um eine leitliniengerechte 
Bundesgesundheitsbl 2020 • 63:322-331 https://doi.org/10.1007/s00103-020-03102-1

(c) Der/die Autor(en) 2020

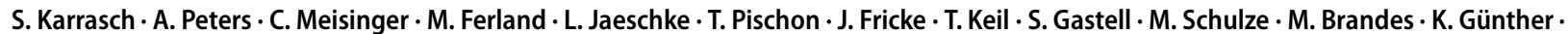
O. Kuß · T. Schikowski · C. Emmel · K.-H. Jöckel · K. B. Michels · C.-W. Franzke · S. Langer · R. Mikolajczyk · A. Jagodzinski · H. Becher · S. Castell · Y. Kemmling · S. Waniek · W. Lieb · K. Wirkner · M. Loeffler · K. H. Greiser · R. Kaaks · N. Legath · K. Berger · S. Schipf · W. Hoffmann · A. Sedlmeier · M. Leitzmann · H. Brenner · B. Holleczek · H. Schulz

\section{Lungenfunktion in der NAKO Gesundheitsstudie: Methoden und erste Ergebnisse}

Zusammenfassung

Hintergrund. Eine Erhebung des respiratorischen Gesundheitszustandes auf Grundlage bundesweit einheitlich durchgeführter Lungenfunktionsmessungen lag in Deutschland bislang nicht vor. Dieser Beitrag beschreibt das Vorgehen bei der Untersuchung der Lungenfunktion in der NAKO Gesundheitsstudie und stellt erste Ergebnisse auf der Datenbasis zur Halbzeit der Basiserhebung vor.

Material und Methoden. Es wurden eine Spirometrie (Level 1) und eine Messung des exhalierten Stickstoffmonoxids (FeNO, Level 2) durchgeführt. Das Qualitätssicherungskonzept beinhaltete regelmäßige Schulungen der Lungenfunktionsprüfung an verschiedenen NAKO-Standorten, Zwischenauswertungen zur Untersuchungsqualität und regelmäßige Kalibrations-/Messkontrollen der Untersuchungsgeräte. Für die Spirometrie wurde zudem ein stufenweises Vorgehen zur Offlinequalitätskontrolle auf Basis der FlussVolumen-Rohkurven etabliert.

Ergebnisse. In den betrachteten Daten $(n=101.734)$ lag eine Spirometrie bei 86.893 Teilnehmenden und eine FeNO-Messung bei 15.228 Teilnehmenden vor. Es fand sich im Mittel ( \pm SD) für die Einsekundenkapazität ( $F E V_{1}$ ) ein Z-Score (gemäß GLI 2012) von $-0,321 \pm 1,047$, für die forcierte Vitalkapazität (FVC) ein Z-Score von -0,153 $\pm 0,941$ und für den Tiffeneau-Index ( $\mathrm{FEV}_{1} / \mathrm{FVC}$ ) ein Z-Score von $-0,337 \pm 0,901$. Die Differenz in $\mathrm{FEV}_{1} / \mathrm{FVC}$ zwischen Rauchern und Nie-Rauchern stieg mit dem Alter an. FeNO lag im geometrischen Mittel bei $14,2 \div 2,0$ ppb, bei aktiven Rauchern war FeNO um $43 \%$ vermindert, bei Nie-/ExRauchern mit respiratorischer Allergie um $16 \%$ erhöht.

Diskussion. Die Ergebnisse der Spirometrie und FeNO-Messungen liegen bezüglich ihrer Verteilungen und bekannter Einflussgrößen im erwarteten Bereich. Die NAKO liefert damit die Datenbasis zur Untersuchung der Atemwegsgesundheit und ihrer Determinanten sowie zur Eruierung der Möglichkeiten zur Prävention respiratorischer Erkrankungen in Deutschland.

Schlüsselwörter

Spirometrie · FeNO - NAKO Gesundheitsstudie . Asthma. COPD

\section{Lung function in the German National Cohort: methods and initial results}

\section{Abstract}

Background. A nationwide assessment of the respiratory status on the basis of standardized lung function measurements has so far not been available in Germany. The present work describes the lung function tests in the German National Cohort (GNC) and presents initial results based on the GNC Midterm Baseline Dataset.

Material and Methods. The assessment of lung function in the GNC comprised spirometry (level 1) and the determination of exhaled nitric oxide (FeNO, level 2). Our quality assurance concept included regular training of lung function test procedures at various GNC sites, interim evaluations of test quality, as well as regular calibration/measurement checks of test equipment. For spirometry, we established a stepwise procedure for offline quality control based on raw flow volume curves.

Results. In the present dataset $(n=101,734)$, spirometry was available for 86,893 study participants and FeNO was available for 15,228 participants. The average $( \pm$ SD) FEV 1 Z score (according to GLI 2012) was $-0.321 \pm 1.047$, the FVC Z score was $-0.153 \pm 0.941$, and the $\mathrm{FEV}_{1} / \mathrm{FVC} Z$ score was $-0.337 \pm 0.901$. The difference in $\mathrm{FEV}_{1} / \mathrm{FVC}$ between current smokers and never-smokers increased with age. The average FeNO was $14.2 \div 2.0 \mathrm{ppb}$.
Current smoking reduced FeNO levels by $43 \%$, whereas respiratory allergy increased FeNO levels by $16 \%$ in nonsmokers.

Discussion. The results of spirometry and the FeNO measurements are in the expected range with regard to their distributions and correlates. The GNC provides a valuable basis for future investigations of respiratory health and its determinants as well as research into the prevention of respiratory diseases in Germany.

Keywords Spirometry - FeNO - German National Cohort . Asthma. COPD
Exspiration innerhalb von drei Ausatemmanövern zu erzielen; dabei wurde ein Messergebnis pro Person aufgezeichnet. Die für ein korrektes und auswertbares Manöver erforderliche gleichmäßige Exspiration über $10 \mathrm{~s}$ bei einer mittleren Ausatemflussrate von $50 \mathrm{~mL} / \mathrm{s}$ wurde vom Messgerät auf Basis der Strömungsdaten sichergestellt, welche während des Manövers online ausgewertet und dem Studienteilnehmenden wie auch dem anleitenden Untersuchenden grafisch unmittelbar rückgemeldet wurden. Im Anschluss wurden die Ergebnisdaten über ein eigens angepasstes Exportprotokoll an das Datenintegrationszentrum der NAKO übertragen.

Um einen gleichbleibend hohen Standard der Lungenfunktionsuntersuchungen zu gewährleisten, wurden umfassende und standardisierte Arbeitsanweisungen für die Lungenfunktionsmessungen und deren Voraussetzungen entwickelt. Alle Lungenfunktionsuntersuchenden innerhalb der NAKO wurden zunächst in ihren jeweiligen Studienzentren in die Grundlagen der Untersuchungsdurchführung eingewiesen und eingelernt. Zur Unterstützung wurden sowohl für die Spirometrie wie auch für die FeNO-Messung eigens Schulungsvideos produziert. Daraufhin wurden die Untersuchenden zentral geschult und zertifiziert sowie regelmäßig rezertifiziert. Zu diesem Zweck wurden regelmäßig Trainingsworkshops an verschiedenen Standorten der NAKO 


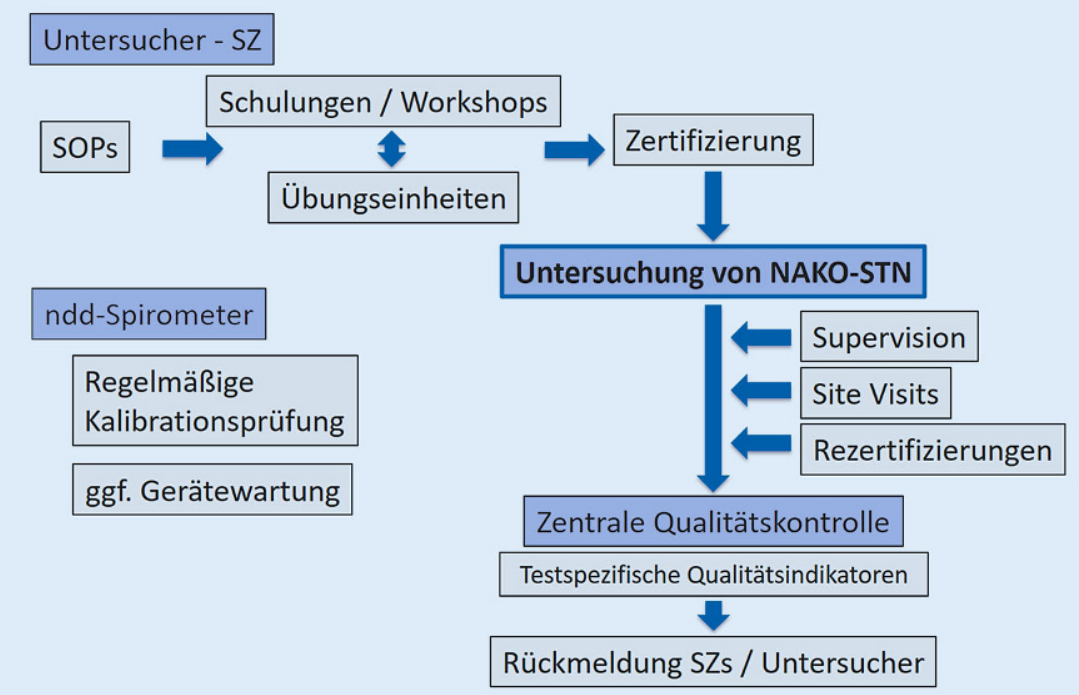

Abb. $1 \Delta$ Qualitätssicherung der Lungenfunktionsuntersuchung während der Feldphase. SOP Standard Operating Procedure, STN Studienteilnehmende, SZ Studienzentrum

Gesundheitsstudie in Abhängigkeit vom jeweiligen regionalen Ausbildungsbedarf organisiert. Im Untersuchungszeitraum erfolgten darüber hinaus Zwischenauswertungen der Untersuchungsqualität der Lungenfunktionsmessungen, deren Ergebnisse den NAKO-Studienzentren untersucherspezifisch zurückgemeldet wurden, um eine gezielte Optimierung zu ermöglichen. Die Konstanz der apparativen Messbedingungen für die Lungenfunktion wurde darüber hinaus für die FeNO-Messung mittels biologischer Kontrollen, d. h. regelmäßiger Selbstmessungen der Untersuchenden des jeweiligen Studienzentrums sichergestellt. Für die Spirometrie erfolgten regelmäßige Kalibrationsprüfungen mittels einer 3-LKalibrationspumpe. Bei Auftreten von zu starken Messwertabweichungen erfolgte eine Gerätewartung durch den jeweiligen Hersteller bzw. ggf. ein Geräteaustausch. Das Vorgehen zur Qualitätssicherung während der Feldphase (2014-2019) ist in $\bullet$ Abb. 1 dargestellt.

Trotz dieses aufwendigen und umfassenden Qualitätssicherungskonzeptes ist auch nach Abschluss der Messungen eine datenbasierte Qualitätskontrolle unerlässlich. Die Spirometrie stellt die Qualitätskontrolle vor besondere Herausforderungen, da eine adäquate Mitarbeit der Teilnehmenden während der forcierten Exspirationsmanöver für ein valides Er- gebnis essenziell ist. Während der Goldstandard für diese Aufgabe eine visuelle Inspektion sämtlicher gewonnener spirometrischer Manöver wäre, ist dieser Ansatz aufgrund der Größe des Datensatzes (im Finaldatensatz sind insgesamt ca. 1.000.000 spirometrische Atemmanöver zu erwarten) nicht realistisch umsetzbar.

Es wurde daher ein stufenweises Vorgehen zur Offlinequalitätskontrolle der spirometrischen Manöver etabliert (- Abb. 2a). Im ersten Schritt erfolgte eine Berechnung der qualitätsrelevanten Kennwerte aus den Fluss-VolumenKurven sowie eine vorläufige Qualitätsbewertung bereits in den NAKOStudienzentren durch den automatischen Algorithmus, der vom Hersteller in die Messsoftware integriert ist. Im zweiten Schritt wurden die gewonnenen Rohkurven aller spirometrischen Manöver einer vertieften computergestützten halbautomatischen Qualitätskontrolle unterzogen. $\mathrm{Zu}$ diesem Zweck wurde eigens eine Auswertesoftware entwickelt, welche jedes Einzelmanöver als entweder akzeptabel, nicht-akzeptabel oder als visuell zu kontrollieren einstuft (• Abb. 2b). Die Manöverbewertung stützt sich dabei auf die vorliegenden Parameter rückextrapoliertes Volumen (BEV), Zeit bis zum Erreichen des Spitzenflusses (PEFT), Gesamtdauer der forcierten Exspiration
(FET) und endexspiratorische Volumenänderung (EOTV). Ohne visuelle Kontrolle verworfen werden Manöver mit PEFT >0,2 sec, Manöver mit BEV zugleich $>0,15 \mathrm{~L}$ und $>5 \%$ der forcierten Vitalkapazität (FVC), Manöver mit EOTV $>0,1 \mathrm{~L}$ bei einer FET von $\leq 6 \mathrm{sec}$ und PEFT $>0,12 \mathrm{sec}$ und Manöver mit FET $\leq 2 \mathrm{sec}$, es sei denn, EOTV ist in diesem Falle $\leq 0,04 \mathrm{~L}$ und PEFT $\leq 0,12 \mathrm{sec}$. Als akzeptabel gewertet werden Manöver mit $\mathrm{BEV}<0,15 \mathrm{~L}$ oder $<5 \%$ der FVC, PEFT $\leq 0,12$ sec und EOTV $\leq 0,04 \mathrm{~L}$ bei einer FET $>2 \mathrm{sec}$. Für die nach diesem Schritt verbleibenden Manöver wird geprüft, ob in der betrachteten Spirometrie bereits akzeptable Manöver vorliegen, die in dem forcierten exspiratorischen Volumen der ersten Sekunde $\left(\mathrm{FEV}_{1}\right)$ und in FVC höhere Werte erbracht haben. Sollte dies zutreffen, so werden die zu prüfenden Manöver ebenfalls verworfen, ansonsten werden sie der visuellen Kontrolle zugeführt.

Abschließend erfolgte eine Einteilung der Spirometrien in die Qualitätskategorien A bis F [27], wobei die Kategorien A und $\mathrm{B}$ die Reproduzierbarkeit von $\mathrm{FEV}_{1}$ und FVC aus mindestens zwei akzeptablen Manövern innerhalb von $\leq 100 \mathrm{~mL}$ (Kategorie A) und $\leq 150 \mathrm{~mL}$ (Kategorie B) anzeigen. Kategorie $\mathrm{C}$ zeigt eine Reproduzierbarkeit von $\leq 200 \mathrm{~mL}$ aus mindestens zwei akzeptablen Manövern an und Kategorie D mindestens ein akzeptables Manöver, das jedoch nicht innerhalb von $\leq 200 \mathrm{~mL}$ reproduzierbar war. In Kategorie F liegt kein akzeptables Manöver vor.

Für die FeNO-Messung wurden die gewonnenen Daten auf Plausibilität und Vollständigkeit geprüft, vereinzelt auftretende Werte $<0 \mathrm{ppb}$ wurden auf $0 \mathrm{ppb}$ gesetzt. Darüber hinaus wurden die Fragebogendaten $\mathrm{zu}$ chronischen und akuten Atemwegssymptomen, diagnostizierten Atemwegserkrankungen und respiratorischer Allergie auf Plausibilität und Vollständigkeit überprüft und auftretende Inkonsistenzen in der Datenbank korrigiert.

Für diese Arbeit wurden die vorliegenden Daten der Spirometrie und FeNO-Messung deskriptiv ausgewertet. Die FeNO-Ergebnisse wurden dabei einerseits nach Raucherstatus (aktiv Rauchende Ja/Nein), andererseits inner- 


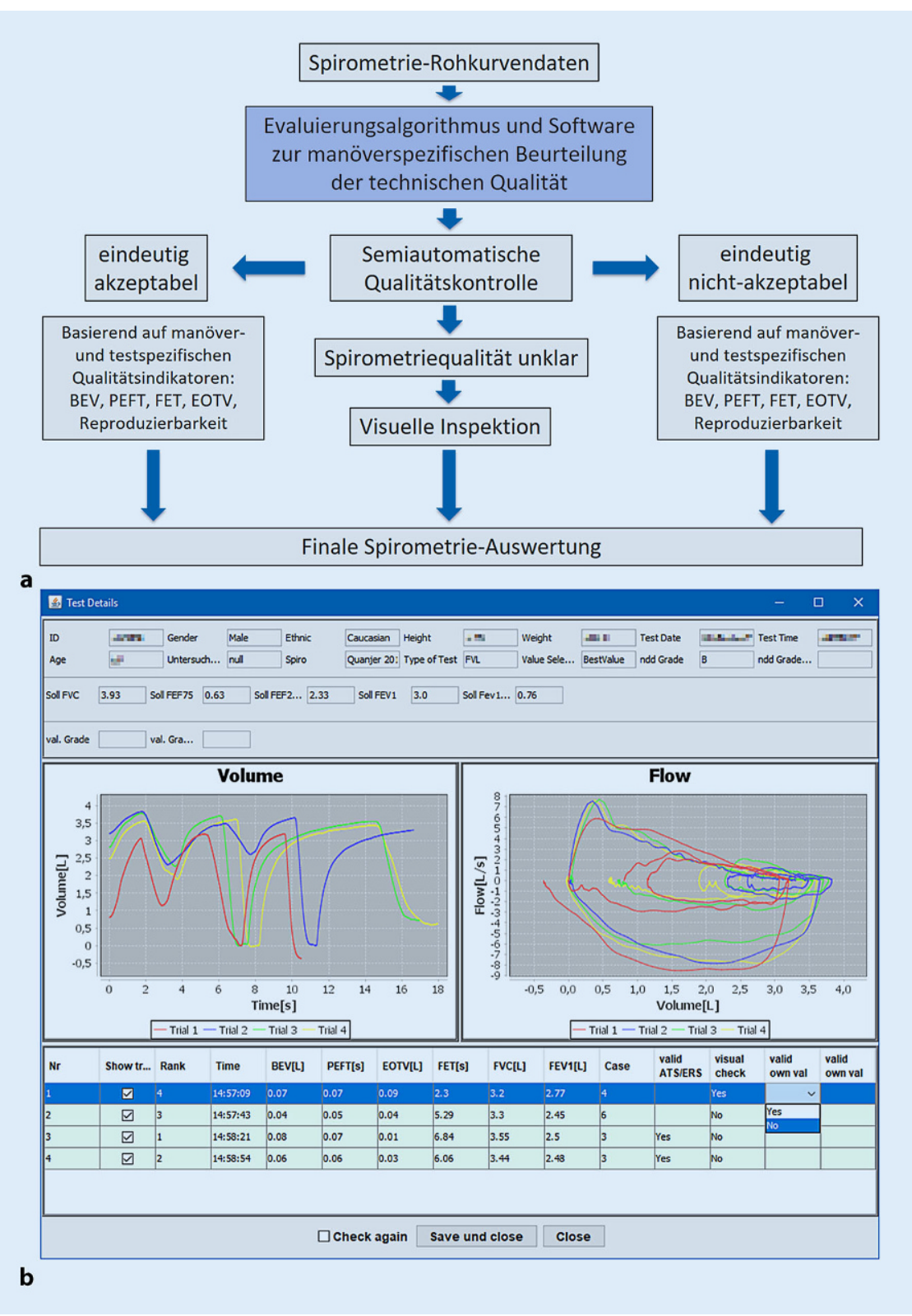

Abb. $2 \Delta$ a Vorgehen bei derQualitätskontrolle der spirometrischen Messdaten. Mittels der entwickelten Auswertesoftware wurden alle gewonnenen Einzelmanöver zunächst basierend auf den Kennwerten BEV, PEFT, FET und EOTV den Kategorien „,eindeutig akzeptabel ", „eindeutig nicht-akzeptabel“ und "Spirometriequalität unklar" zugeordnet. Atemmanöver unklarer Qualität wurden daraufhin der visuellen Kontrolle zugeführt. b Screenshot der entwickelten Auswertesoftware. Im oberen Teil werden anthropometrische Daten der Studienteilnehmenden, individuelle spirometrische Sollwerte sowie testbezogene Daten angegeben. Darunter werden auf Basis der Rohkurvendaten die Fluss-Volumenund Volumen-Zeit-Kurven sämtlicher gewonnener Atemmanöver in verschiedenen Farben dargestellt. Im unteren Teil sind qualitätsrelevante Kennwerte sowie wichtige spirometrische Indizes für alle Einzelmanöver in tabellarischer Form aufgeführt. Die Bewertung der Einzelmanöver erfolgt in dieser Tabelle über eine manöverspezifische Schaltfläche. BEV rückextrapoliertes Volumen, EOTV endexspiratorische Volumenänderung, FETGesamtdauer der forcierten Exspiration, PEFTZeit bis zum Erreichen des Spitzenflusses halb der Nichtrauchenden (Nie- und Ex-Rauchende) nach Vorliegen einer respiratorischen Allergie (hier definiert als Vorliegen von Asthma, Heuschnupfen oder Symptomen einer allergischen Rhinitis) stratifiziert beschrieben. Die spirometrischen Ergebnisse für $\mathrm{FEV}_{1}$, FVC und den Quotienten $\mathrm{FEV}_{1} / \mathrm{FVC}$ (klassisch Tiffeneau-Wert genannt) von Teilnehmenden mit mindestens einem gültigen Spirometriemanöver wurden unter Berücksichtigung von Alter, Geschlecht, Körpergröße und Ethnizität auf die aktuellen Referenzwerte der Global Lung Function Initiative bezogen [28] und Ergebnisse der auf diese Weise gewonnenen Z-Scores einerseits nach verwendeten Geräten und Studienzentren aufgeschlüsselt, andererseits nach 5-Jahres-Altersgruppen sowie Raucherstatus (aktiv Rauchende, Ex-Rauchende, Nie-Rauchende). Die Auswertungen erfolgten mit der Software SAS 9.4 (SAS Institute, Cary, NC, USA) und Statgraphics Centurion XVII (Statpoint Technologies Inc., Warrenton, VA, USA).

\section{Ergebnisse}

Die für die vorliegende Arbeit verwendete Datenbasis zur Halbzeit der NAKOBasiserhebung umfasste 101.734 Studienteilnehmende im Alter zwischen 20 und 75 Jahren (der Anteil der Teilnehmenden $\geq 70$ Jahre war dabei $2,9 \%$ ).

Spirometrische Messwerte des Level1-Programms lagen von 86.893 Studienteilnehmenden (54,2\% Frauen) vor. Die Auswertung sämtlicher Atemmanöver mittels der eigens entwickelten Software erbrachte für 57.514 Spirometrien eine automatische Qualitätsbewertung, bei 29.379 Spirometrien erfolgte eine visuelle Beurteilung. Die Ergebnisse der Qualitätsbewertung über die einzelnen Studienzentren (SZ) der NAKO hinweg sind in $\square$ Abb. 3 dargestellt. Dabei zeigte sich ein Gesamtanteil von $96,0 \%$ der Spirometrien mit mindestens einem gültigen Manöver (93,4-97,7\% über die Studienzentren hinweg) und 70,4\% der Spirometrien in den Kategorien A oder B $(57,0-78,5 \%$ über die Studienzentren hinweg).

In einer Auswertung über alle Studienzentren hinweg zeigten sich in $\mathrm{FEV}_{1}$, FVC 


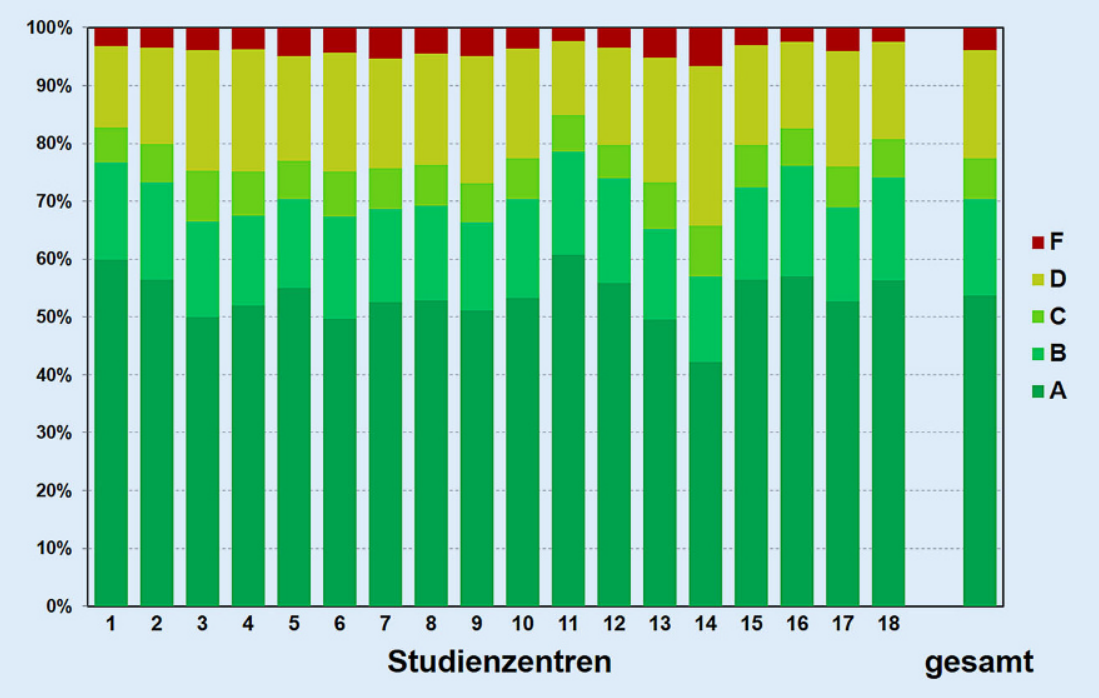

Abb. $3 \Delta$ Qualitätsgrade der Spirometrien aufgeschlüsselt nach NAKO-Studienzentren sowie über die gesamte Datenbasis zur Halbzeit der Basiserhebung. Dargestellt sind die prozentualen Anteile der fünf Qualitätskategorien A bis F an den im jeweiligen Studienzentrum bzw. im Gesamtdatensatz vorliegenden Spirometrien. A: mindestens zwei akzeptable Manöver mit einer Reproduzierbarkeit von $\mathrm{FEV}_{1}$ und FVC innerhalb von $\leq 100 \mathrm{~mL}, \mathrm{~B}$ : mindestens zwei akzeptable Manöver mit einer Reproduzierbarkeit von $\mathrm{FEV}_{1}$ und $\mathrm{FVC}$ innerhalb von $\leq 150 \mathrm{~mL}$, C: mindestens zwei akzeptable Manöver mit einer Reproduzierbarkeit von FEV ${ }_{1}$ und FVC innerhalb von $\leq 200 \mathrm{~mL}$, D: mindestens ein akzeptables Manöver, das jedoch nicht für $\mathrm{FEV}_{1}$ und FVC innerhalb von $\leq 200 \mathrm{~mL}$ reproduzierbar war, F: kein akzeptables Manöver

und $\mathrm{FEV}_{1} / \mathrm{FVC}$ mittlere Ergebniswerte ( \pm Standardabweichung) für den $\mathrm{FEV}_{1^{-}}$ Z-Score von $-0,321 \pm 1,047$ (Frauen: $-0,276 \pm 1,040$, Männer: $-0,375 \pm 1,054$ ), für den FVC-Z-Score von $-0,153 \pm 0,941$ (Frauen: $-0,089 \pm 0,937$, Männer: $-0,229 \pm 0,939)$ und für den $\mathrm{FEV}_{1} / \mathrm{FVC}-$ Z-Score von $-0,337 \pm 0,901$ (Frauen: $-0,380 \pm 0,855$, Männer: $-0,287 \pm 0,950$ ), in einer Auswertung nach Messgeräten fanden sich daneben keine klinisch bedeutsamen Abweichungen zwischen den Geräten (『Abb. 4). In der nach Raucherstatus und Altersgruppen stratifizierten Auswertung zeigte sich erwartungsgemäß eine über den betrachteten Altersbereich hinweg deutlichere Reduktion von $\mathrm{FEV}_{1} / \mathrm{FVC}$ bei aktiv Rauchenden gegenüber Ex-Rauchenden sowie bei ExRauchenden gegenüber Nie-Rauchenden. Interpretiert man versuchsweise den Quotienten $\mathrm{FEV}_{1} / \mathrm{FVC}$ als $\mathrm{Maß}$ für das "spirometrische Alter" der Lunge [29], so ergibt sich daraus bei aktiv Rauchenden eine „spirometrische $\mathrm{Al}$ tersdifferenz" von etwa 5 Jahren gegenüber Nie-Rauchenden im Lebensalter von 25-29 Jahren und eine Differenz

\section{Diskussion}

Die Lungenfunktionsmessungen im Rahmen der NAKO Gesundheitsstudie bieten eine einzigartige Möglichkeit, in großem Maßstab Atemwegsbeschwerden und Lungenerkrankungen auf Basis standardisierter, qualitativ hochwertiger funktioneller Daten zu untersuchen, und ermöglichen Analysen von Determinanten des respiratorischen Funktionsstatus auf bundesweiter Ebene. Das breite Untersuchungsspektrum im Rahmen der NAKO bietet darüber hinaus die Möglichkeit, den Zusammenhang von Atemwegserkrankungen und Begleitkrankheiten jenseits der Atemwege zu untersuchen.

Da die NAKO auf der Allgemeinbevölkerung basiert, schließt sie die wichtige Personengruppe mit diskreten, jedoch noch nicht klinisch manifesten funktionellen respiratorischen Beeinträchtigungen ein, die in klinischen Kohorten aufgrund der jeweiligen Einschlusskriterien nicht oder nicht in hinreichendem Maße berücksichtigt ist. Die Identifikation dieser Personen und ihrer möglichen Charakteristika ist von großer Bedeutung als Adressaten für gezielte Präventionsmaßnahmen, um nach Möglichkeit ein Fortschreiten respiratorischer Beeinträchtigung zu manifesten Lungenerkrankungen zu verhindern, für die bislang kaum im eigentlichen Sinne kurative therapeutische Ansätze zur Verfügung stehen.

\section{Hauptergebnisse und Vergleich mit anderen Studien}

Die Spirometrien in der betrachteten $\mathrm{Da}$ tenbasis zur Halbzeit der NAKO-Basiserhebung sind sowohl insgesamt als auch in den einzelnen Studienzentren von erfreulich hoher Qualität. Der beobachtete Anteil von $>70 \%$ der Spirometrien in den Qualitätskategorien A und B, in welchen sich die Reproduzierbarkeitsanforderungen der ATS/ERS-SpirometrieLeitlinien abbilden [24], sowie von $>95 \%$ der Spirometrien mit auswertbaren Ergebniskurven ist vergleichbar mit Beobachtungen anderer großer Erhebungen [30-32], wenngleich naturgemäß etwas niedriger als in spezifischen Studien zu Lungengesundheit oder -erkrankungen 


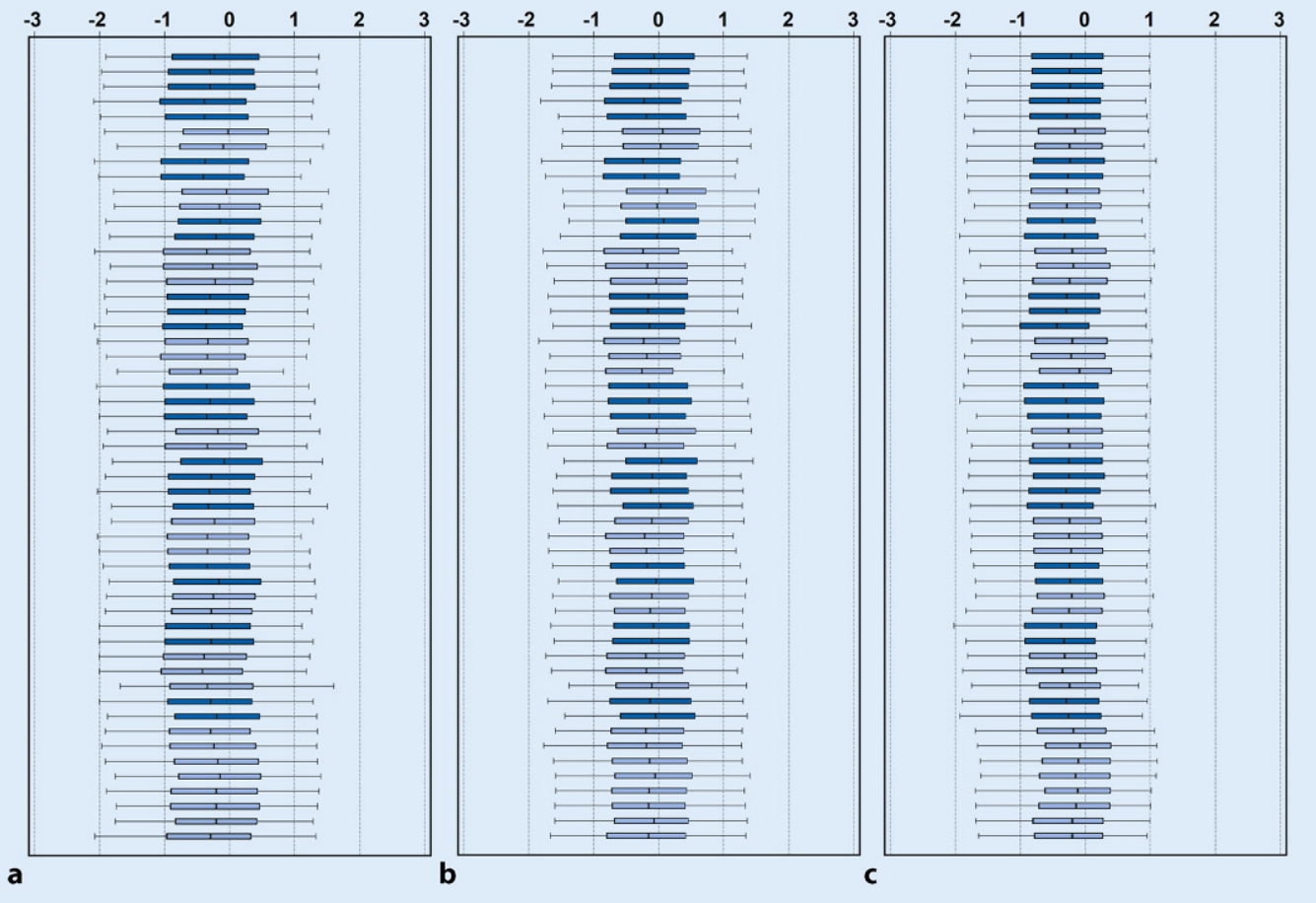

Abb. $4<$ Ergebnisse für a $\mathrm{FEV}_{1}, \mathrm{~b}$ FVC und c FEV $1 / F V C$ (als Z-Scores gemäß den Sollwerten der Global Lung Function Initiative) aufgeschlüsselt nach Messgeräten (Einzelbalken) und Studienzentren (Farben wechselnd zwischen dunkelblau und hellblau). Boxplots mit Median, 25. und 75. Perzentile (Whisker zeigen 5. und 95. Perzentile). $F E V_{1}$ forciertes exspiratorisches Volumen der ersten Sekunde, FVC forcierte Vitalkapazität FEV $1 /$ FVC Tiffeneau-Index

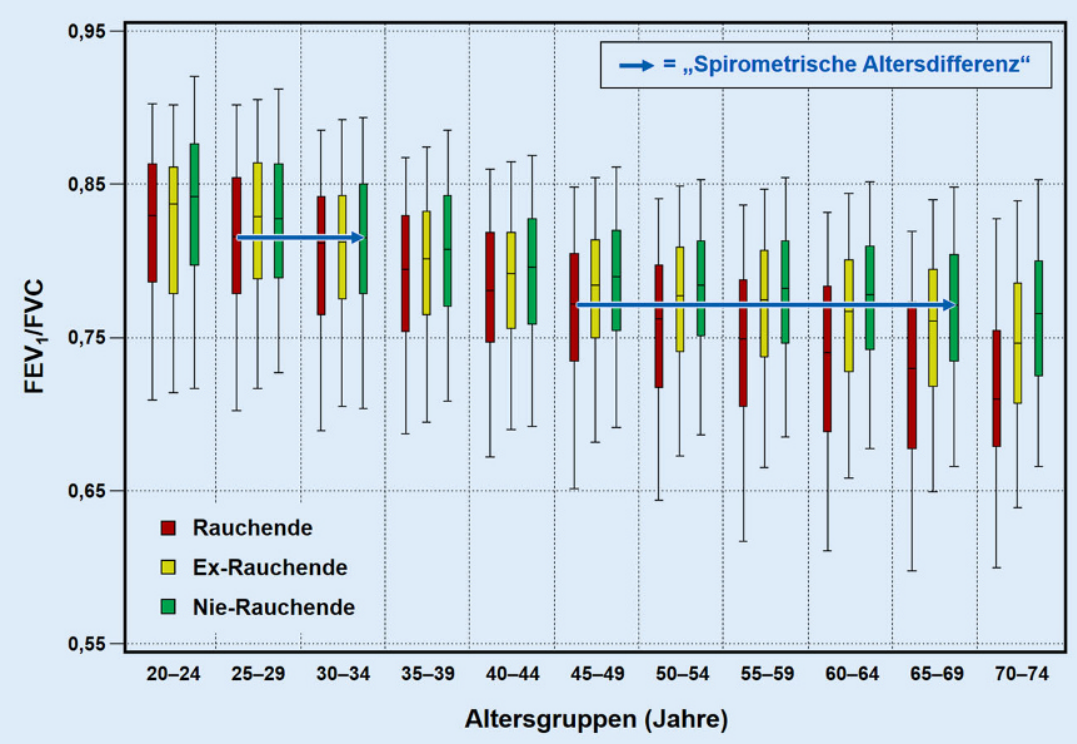

Abb. $5 \Delta$ Ergebnisse für $\mathrm{FEV}_{1} / \mathrm{FVC}$ nach Raucherstatus über den betrachteten Altersbereich von 20-75 Jahren. Rot Rauchende, gelb Ex-Rauchende, grün Nie-Rauchende. Boxplots mit Median, 25. und 75. Perzentile (Whisker zeigen 5. und 95. Perzentile). FEV/FVC Tiffeneau-Index. Erläuterungen siehe Text

$[33,34]$. Insbesondere der geringe Anteil von lediglich 4,0\% der Spirometrien ohne ein mit hinreichender Verlässlichkeit auswertbares Exspirationsmanöver führt zu einem erfreulich umfassenden Datensatz. Angesichts des erheblichen Datenumfangs der NAKO Gesundheitsstudie ermöglicht dabei die zur halbautomatischen Qualitätsbewertung entwickelte Auswertesoftware eine Fokussierung der visuellen Rohkurvenbeurteilung auf jene Spirometrien, deren Qualität auf Basis der primären manöverspezifischen Qualitätsindikatoren nicht hinreichend ver- lässlich zu bewerten ist. Damit ist zwar dennoch für rund ein Drittel der gewonnenen Spirometrien (33,8\%) eine visuelle Kontrolle erforderlich, es zeigte sich jedoch in dieser ersten Anwendung des Vorgehens, dass der daraus resultierende, wenn auch erhebliche Aufwand der Offlinequalitätskontrolle im Studiensetting einer so großen Gesundheitsstudie realistisch umsetzbar ist. Ob möglicherweise durch die Weiterentwicklung des Algorithmus, etwa durch eine Verringerung der Toleranzbereiche für die qualitätsrelevanten Kennwerte oder den Einschluss neuer Kennwerte, eine Effizienzsteigerung ohne einen als relevant zu betrachtenden Qualitätsverlust zu erzielen ist, wird bei Vorliegen des Gesamtdatensatzes der Baselineerhebung in weitergehenden Analysen eruiert werden können.

Die beobachteten Ergebnisse sowohl für spirometrische Volumina und Tiffeneau-Index als auch für FeNO liegen hinsichtlich Mittelwert und Verteilungen im erwarteten Bereich. Die gegenüber den GLI-Referenzwerten [28] im Mittel leicht negativen Werte für $\mathrm{FEV}_{1}-\mathrm{Z}$ Score, FVC-Z-Score und FEV ${ }_{1} /$ FVC-ZScore erklären sich dabei aus der Tatsache, dass in die vorliegende Analyse auch aktiv Rauchende und Ex-Rauchen- 

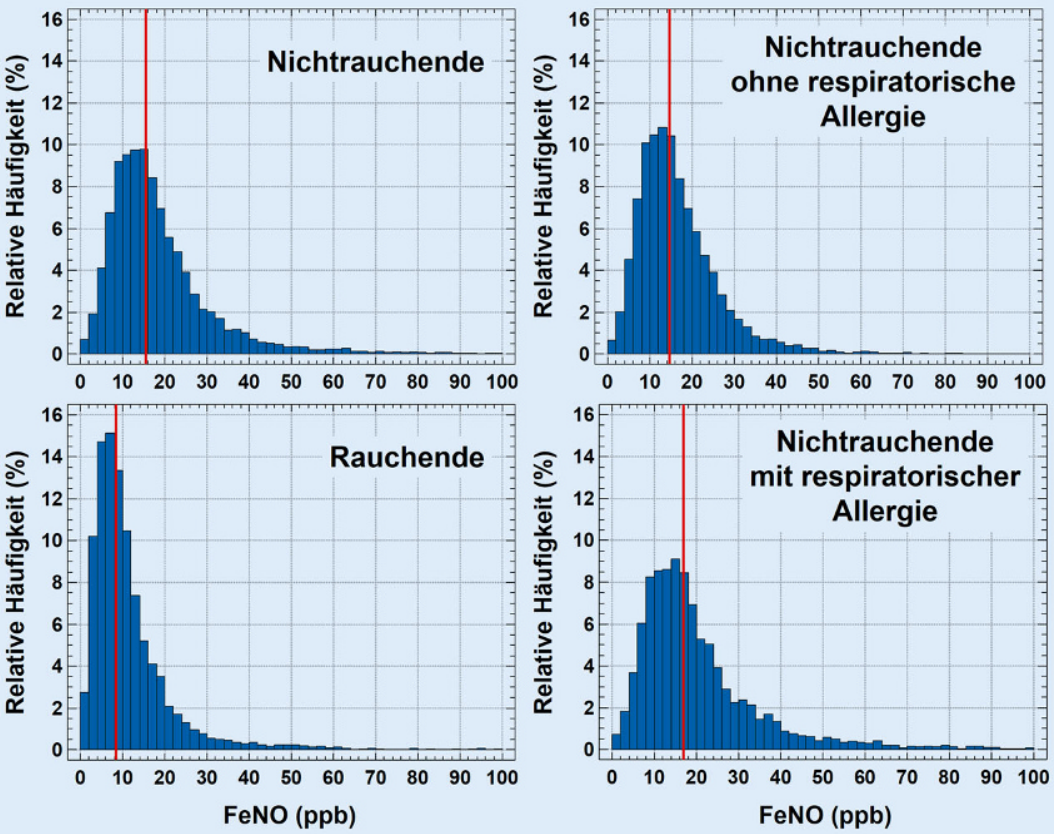

Abb. 6 \ Häufigkeitsverteilungen für FeNO in Abhängigkeit vom Raucherstatus und Vorliegen einer respiratorischen Allergie (innerhalb der Nie- und Ex-Rauchenden, $n=11.909$ ). Rot geometrische Mittelwerte. FeNO Anteil von Stickstoffmonoxid in der Ausatemluft, $p p b$ „parts per billion“

de sowie Patienten mit Lungenerkrankungen eingeschlossen wurden. Die jeweils nahe dem Wert 1,0 liegenden Ergebnisse für die Standardabweichung der Z-Scores in allen drei Parametern weisen zudem darauf hin, dass auch diese Verteilungseigenschaft mit der GLI-Referenz gut in Einklang steht. Im Hinblick auf das bei aktiv Rauchenden beobachtete höhere ,spirometrische Alter “gegenüber Ex- und Nie-Rauchenden ist zu beachten, dass sich daraus nicht unmittelbar auf eine in vergleichbarer Weise als vorzeitig gealtert imponierende Einschränkung in anderen Funktionseinheiten des respiratorischen Systems rückschließen lässt [29]. Dies wäre in weiteren Analysen, etwa unter Berücksichtigung struktureller Charakteristika aus der Bildgebung, zu eruieren.

Im Hinblick auf FeNO stehen die hier ermittelten Effekte aktiven Rauchens sowie einer vorliegenden respiratorischen Allergie auf das Messergebnis in guter Übereinstimmung mit den Daten aus vorliegenden Untersuchungen [35-37]. Dabei lag der Effekt des Rauchens mit $-43 \%$ eher im oberen Bereich des berichteten Spektrums, der Effekt einer respiratorischen Allergie mit

\section{Forschungsperspektiven}

Perspektivisch ermöglichen die vorliegenden vollständigen spirometrischen Rohdaten von Fluss und Volumen jedes einzelnen forcierten Exspirationsmanövers auch die Identifizierung neuer Parameter, die aus spirometrischen FlussVolumen-Kurven abgeleitet werden können, entweder für den Einsatz in der Weiterentwicklung der hier beschriebenen halbautomatischen Qualitätskontrolle oder bei der klinischen Interpretation der Manöver.

Ein im Rahmen der NAKO-Untersuchungen in einer weiteren Teilstichprobe von 30.000 Teilnehmenden zusätzlich durchgeführter MRT-Ganzkörperscan, auch wenn er nicht spezifisch auf die Erfassung der Lunge zielt, kann darüber hinaus die Möglichkeit eröffnen, den Zusammenhang zwischen strukturellen und funktionellen Veränderungen der kardiorespiratorischen Einheit und Einflüssen von Umwelt- und Lebensstilfaktoren zu untersuchen.

Daneben erlaubt der Datensatz der NAKO auch die Untersuchung möglicher Unterschiede in der Lungenfunktion zwischen verschiedenen geografischen Regionen oder in Hinblick auf den Urbanisierungsgrad. Dies schließt die Beurteilung der geschätzten Gesundheitskosten im Zusammenhang mit Atemwegserkrankungen und der jeweiligen Inanspruchnahme des Gesundheitssystems ein. Es konnte bereits in eigenen früheren Arbeiten gezeigt werden, dass insbesondere COPD mit erheblichen Gesundheitskosten verbunden ist [39] und dass auch bei Patienten mit diskreten Lungenfunktionseinschränkungen, die häufig noch nicht ärztlich diagnostiziert sind, eine geringere gesundheitsbezogene Lebensqualität vorliegt [40]. Angesichts einer vorläufigen mittleren Responsequote von rund $18 \%$ in der NAKO ist bei der bundesweiten Beschreibung des erhobenen respiratorischen Status prinzipiell ein möglicher Selektionsbias durch Nichtteilnahme in Betracht zu ziehen, für die Variablen Alter und Geschlecht wurde allerdings bereits stratifiziert rekrutiert. Am Ende der Baselineerhebung werden zudem zusätzliche Informationen bezüglich der 
Non-Responder berücksichtigt werden können. Im Hinblick auf die Anlage der NAKO als Langzeitstudie liegt der Schwerpunkt der Betrachtung darüber hinaus grundsätzlich vorwiegend auf der internen Validität [20]. Von besonderer Bedeutung sind vor diesem Hintergrund auch die bereits begonnenen Follow-upUntersuchungen der Lungenfunktion in der NAKO, auf deren Basis Personen mit einer beschleunigten Abnahme in respiratorischen Messgrößen auch oberhalb der diagnostischen Grenzwerte einer Funktionseinschränkung identifiziert werden können.

\section{Schlussfolgerung}

Sobald die Lungenfunktionsdaten der Baselineerhebung der NAKO vollständig vorliegen, können diese nicht nur als Basis für eine Vielzahl von Forschungsfragen im Hinblick auf die Quantifizierung der Zusammenhänge etwa von Umwelteinflüssen und Atemwegsgesundheit dienen, sondern auch als Grundlage einer evidenzbasierten Orientierung für nationale wie regionale Entscheidungen hinsichtlich der Prävention und medizinischen Versorgung respiratorischer Erkrankungen in Deutschland.

\section{Korrespondenzadresse}

\section{Dr. med. Stefan Karrasch}

Institut und Poliklinik für Arbeits-, Sozial- und Umweltmedizin, Klinikum der Universität München

Ziemssenstr. 1, 80336 München, Deutschland stefan.karrasch@med.uni-muenchen.de

Danksagung. Wir danken allen Teilnehmerinnen und Teilnehmern der NAKO Gesundheitsstudie sowie den Mitarbeiterinnen und Mitarbeitern der NAKO Gesundheitsstudie.

Förderung. Dieses Projekt wurde mit Daten der NAKO Gesundheitsstudie durchgeführt (www.nako. de). Die NAKO Gesundheitsstudie wird durch das Bundesministerium für Bildung und Forschung (BMBF, Förderkennzeichen 01ER1301A/B/C und 01ER1511D), die Bundesländer und die HelmholtzGemeinschaft gefördert sowie durch die beteiligten Universitäten und Institute der Leibniz-Gemeinschaft finanziell unterstützt.

Funding. Open Access funding provided by Projekt DEAL.

\section{Einhaltung ethischer Richtlinien}

Interessenkonflikt. S. Karrasch, A. Peters, C. Meisinger, M. Ferland, L. Jaeschke, T. Pischon, J. Fricke, T. Keil, S. Gastell, M. Schulze, M. Brandes, K. Günther, O. Kuß, T. Schikowski, C. Emmel, K.-H. Jöckel, K.B. Michels, C.-W. Franzke, S. Langer, R. Mikolajczyk, A. Jagodzinski, H. Becher, S. Castell, Y. Kemmling, S. Waniek, W. Lieb, K. Wirkner, M. Loeffler, K.H. Greiser, R. Kaaks, N. Legath, K. Berger, S. Schipf,W. Hoffmann, A. Sedlmeier, M. Leitzmann, H. Brenner, B. Holleczek und H. Schulz geben an, dass kein Interessenkonflikt besteht.

Alle beschriebenen Untersuchungen wurden mit Zustimmung der zuständigen Ethik-Kommissionen, im Einklang mit nationalem Recht sowie gemäß der Deklaration von Helsinki von 1975 (in der aktuellen, überarbeiteten Fassung) durchgeführt. Von allen Teilnehmenden liegt eine Einverständniserklärung vor.

Open Access. Dieser Artikel wird unter der Creative Commons Namensnennung 4.0 International Lizenz veröffentlicht, welche die Nutzung, Vervielfältigung, Bearbeitung, Verbreitung und Wiedergabe in jeglichem Medium und Format erlaubt, sofern Sie den/die ursprünglichen Autor(en) und die Quelle ordnungsgemäß nennen, einen Link zur Creative Commons Lizenz beifügen und angeben, ob Änderungen vorgenommen wurden.

Die in diesem Artikel enthaltenen Bilder und sonstiges Drittmaterial unterliegen ebenfalls der genannten Creative Commons Lizenz, sofern sich aus der Abbildungslegende nichts anderes ergibt. Sofern das betreffende Material nicht unter der genannten Creative Commons Lizenz steht und die betreffende Handlung nicht nach gesetzlichen Vorschriften erlaubt ist, ist für die oben aufgeführten Weiterverwendungen des Materials die Einwilligung des jeweiligen Rechteinhabers einzuholen.

Weitere Details zur Lizenz entnehmen Sie bitte der Lizenzinformation auf http://creativecommons.org/ licenses/by/4.0/deed.de.

\section{Literatur}

1. WHO (2014) Global status report on noncommunicable diseases

2. ERS (2013) European lung white book

3. WHO (2010) High-level consultation in the European region on noncommunicable diseases

4. De Matteis S, Jarvis D, Hutchings S et al (2016) Occupations associated with COPD risk in the large population-basedUKbiobank cohortstudy. Occup Environ Med 73:378-384

5. Anderson M, Qvarfordt M, Svartengren M (2016) Spirometryin the 5000 subjects of the lifegene pilot study in Sweden: evaluating different reference equations, BMI and thorax measurements. Eur Respir J 48:PA2247

6. de Jong K, Boezen HM, Kromhout H et al (2014) Pesticides and other occupational exposures are associated with airway obstruction: the lifelines cohort study. Occup Environ Med 71:88-96

7. Awadalla P, Boileau C, Payette Y et al (2013) Cohort profile of the CARTaGENE study: quebec's population-based biobank for public health and personalized genomics. Int J Epidemiol 42:1285-1299
8. Tilert T, Dillon C, Paulose-Ram R, Hnizdo E, Doney B (2013) Estimating the U.S. prevalence of chronic obstructive pulmonary disease using preand post-bronchodilator spirometry: the national health and nutrition examination survey (NHANES) 2007-2010. Respir Res 14:103

9. Cerveri I, Accordini S, Verlato G et al (2001) Variations in the prevalence across countries of chronic bronchitis and smoking habits in young adults. Eur Respir J 18:85-92

10. Gibson GJ, Loddenkemper R, Sibille $Y$, Lundback B (2013) Respiratory health and disease in Europe: the new European lung white book. Eur Respir J 42(3):559-563. https://doi.org/10.1183/ 09031936.00105513

11. Gillissen A, Welte T (2014) Weißbuch Lunge 2014. Herausforderungen - Zukunftsperspektiven Forschungsansätze. Zur Lage und Zukunft der Pneumologie in Deutschland. Frischtexte Verlag, Herne

12. Gosswald A, Lange M, Kamtsiuris P, Kurth BM (2012) DEGS: German health interview and examination survey for adults. A nationwide cross-sectional and longitudinal study within the framework of health monitoring conducted by the Robert Koch institute. Bundesgesundheitsblatt Gesundheitsforschung Gesundheitsschutz 55:775-780

13. Robert Koch-Institut (2014) Daten und Fakten: Ergebnisse der Studie "Gesundheit in Deutschland aktuell 2012". RKI, Berlin

14. Holle R, Happich M, Lowel H, Wichmann HE, MONICA/KORA Study Group (2005) KORA-a research platform for population based health research. Gesundheitswesen 67(1):S19-25

15. Ewert R, Ittermann T, Bollmann T et al (2017) Lung health data of the study of health in Pomerania-a review of samples, methods and first results. Pneumologie 71:17-35

16. HCHS (Hamburg City Health Study) (2019) Gezielt vorbeugen, gesünder leben. http://hchs. hamburg/fuer-morgen/ziele/. Zugegriffen: 29. Sept. 2019

17. Karch A, Vogelmeier C, Welte T et al (2016) The German COPD cohort COSYCONET: aims, methods and descriptive analysis of the study population at baseline. Respir Med 114:27-37

18. Kardos P, Vogelmeier C, Buhl R, Criee CP, Worth H (2015) The prospective non-Interventional DACCORD study in the national COPD registry in Germany: design and methods. BMC Pulm Med 15:2

19. Fuchs $O$, Bahmer T, Weckmann M, Dittrich A-M, Schaub B, Rösler B et al (2018) The all age asthma cohort (ALLIANCE) - from early beginnings to chronic disease: a longitudinal cohort study. BMC Pulm Med 18(1)

20. Schipf S, Schöne G, Schmidt B et al (2020) Die Basiserhebung der NAKO Gesundheitsstudie: Teilnahme an den Untersuchungsmodulen, Qualitätssicherung und Nutzung von Sekundärdaten. Bundesgesundheitsblatt Gesundheitsforschung Gesundheitsschutz. https://doi.org/10. 1007/s00103-020-03093-z

21. Criee CP, Baur X, Berdel D et al (2015) Standardization of spirometry: 2015 update. Published by German Atemwegsliga, German respiratory society and German society of occupational and environmental medicine. Pneumologie 69:147-164

22. ATS/ERS (2005) ATS/ERS Recommendations for Standardized Procedures for the Online and Offline Measurement of Exhaled Lower Respiratory Nitric Oxide and Nasal Nitric Oxide, 2005. Am J Resp Crit Care 171(8):912-930 
23. Dweik RA, Boggs PB, Erzurum SC et al (2011) An official ATS clinical practice guideline: interpretation of exhaled nitric oxide levels (FENO) for clinical applications. Am J Respir Crit Care Med 184:602-615

24. Miller MR, Hankinson J, Brusasco V et al (2005) Standardisation of spirometry. Eur Respir J 26:319-338

25. Coates AL, Graham BL, McFadden RG et al (2013) Spirometry in primary care. Can Respir J 20:13-21

26. Cooper BG (2011) An update on contraindications for lung function testing. Thorax 66:714-723

27. Ferguson GT, Enright PL, Buist AS, Higgins MW (2000) Office spirometry for lung health assessment in adults: a consensus statement from the national lung health education program. Chest 117:1146-1161

28. Quanjer PH, Stanojevic S, Cole TJ et al (2012) Multiethnic reference values for spirometry for the 3-95-yr age range: the global lung function 2012 equations. Eur Respir J 40:1324-1343

29. Karrasch S, Behr J, Huber RM et al (2018) Heterogeneous pattern of differences in respiratory parameters between elderly with either good or poor FEV1. BMC Pulm Med 18:27

30. Enright PL, Skloot GS, Cox-Ganser JM, Udasin IG, Herbert R (2010) Quality of spirometry performed by 13,599 participants in the world trade center worker and volunteer medical screening program. Respir Care 55:303-309

31. Maio S, Sherrill DL, MacNee W et al (2012) The European respiratory society spirometry tent: a unique form of screening for airway obstruction. EurRespir J39:1458-1467

32. Oelsner EC, Balte PP, Cassano PA et al (2018) Harmonization of respiratory data from 9 US population-based cohorts: the NHLBI pooled cohorts study. Am JEpidemiol 187:2265-2278

33. Perez-Padilla R, Vazquez-Garcia JC, Marquez MN, Menezes AM, Group P (2008) Spirometry qualitycontrol strategies in a multinational study of the prevalence of chronic obstructive pulmonary disease. Respir Care 53:1019-1026

34. Enright P, Vollmer WM, Lamprecht B et al (2011) Quality of spirometry tests performed by 9893 adults in 14 countries: the BOLD study. Respir Med 105:1507-1515

35. Karrasch S, Ernst K, Behr J et al (2011) Exhaled nitric oxide and influencing factors in a random population sample. Respir Med 105:713-718

36. Dressel H, de la Motte D, Reichert J et al (2008) Exhaled nitric oxide: independent effects of atopy, smoking, respiratory tract infection, gender and height. Respir Med 102:962-969

37. Olin AC, Rosengren A, Thelle DS, Lissner L, Toren K (2010) Increased fraction of exhaled nitric oxide predicts new-onset wheeze in a general population. Am J Respir Crit Care Med 181:324-327

38. Karrasch S, Linde K, Rucker G et al (2017) Accuracy of FENO for diagnosing asthma: a systematic review. Thorax 72:109-116

39. Wacker ME, Jorres RA, Schulz H et al (2016) Direct and indirect costs of COPD and its comorbidities: results from the German COSYCONET study. Respir Med 111:39-46

40. Wacker ME, Hunger M, Karrasch S et al (2014) Health-related quality of life and chronic obstructive pulmonary disease in early stages-longitudinal results from the populationbased KORA cohort in a working age population. BMC Pulm Med 14:134

\section{Affiliations}

'Institut für Epidemiologie, Helmholtz Zentrum München, München, Deutschland; ${ }^{2}$ Institut und Poliklinik für Arbeits-, Sozial- und Umweltmedizin, Klinikum der Universität München, München, Deutschland; ${ }^{3}$ Comprehensive Pneumology Center Munich (CPC-M), Mitglied des Deutschen Zentrums für Lungenforschung (DZL), München, Deutschland; ${ }^{4}$ SFG Klinische Epidemiologie, Helmholtz Zentrum München, Neuherberg, Deutschland; ${ }^{5}$ Lehrstuhl für Epidemiologie, am UNIKA-T Augsburg, LudwigMaximilians-Universität München, Augsburg, Deutschland; ${ }^{6}$ Forschergruppe Molekulare Epidemiologie, Max-Delbrück-Centrum für Molekulare Medizin in der Helmholtz-Gemeinschaft (MDC), Berlin, Deutschland; ${ }^{7}$ Charité - Universitätsmedizin Berlin, Berlin, Deutschland; ${ }^{8}$ Deutsches Zentrum für Herz Kreislauf-Forschung (DZHK), Partnerstandort Berlin, Deutschland; ${ }^{9} \mathrm{MDC} /$ BIH Biobank, Max-DelbrückCentrum für Molekulare Medizin in der Helmholtz-Gemeinschaft (MDC) und Berlin Institute of Health (BIH), Berlin, Deutschland; ${ }^{10}$ Institut für Sozialmedizin, Epidemiologie und Gesundheitsökonomie, Charité - Universitätsmedizin Berlin, Berlin, Deutschland; "Institut für Klinische Epidemiologie und Biometrie, Universität Würzburg, Würzburg, Deutschland; ${ }^{12}$ Landesinstitut für Gesundheit, Bayerisches Landesamt für Gesundheit und Lebensmittelsicherheit, Bad Kissingen, Deutschland; ${ }^{13}$ Zentrale Einrichtung NAKO Studienzentrum, Deutsches Institut für Ernährungsforschung, Potsdam-Rehbrücke, Deutschland; ${ }^{14}$ Abteilung Molekulare Epidemiologie, Deutsches Institut für Ernährungsforschung, Potsdam-Rehbrücke, Deutschland; ${ }^{15}$ Leibniz-Institut für Präventionsforschung und Epidemiologie - BIPS, Bremen, Deutschland; ${ }^{16}$ Institut für Biometrie und Epidemiologie, Deutsches Diabetes-Zentrum (DDZ), LeibnizZentrum für Diabetes-Forschung an der Heinrich-Heine-Universität Düsseldorf, Düsseldorf, Deutschland; ${ }^{17}$ IUF - Leibniz-Institut für umweltmedizinische Forschung Düsseldorf, Düsseldorf, Deutschland; ${ }^{18}$ Institut für Medizinische Informatik, Biometrie und Epidemiologie (IMIBE), Universitätsklinikum Essen, Universität Duisburg-Essen, Essen, Deutschland; ${ }^{19}$ Institut für Prävention und Tumorepidemiologie, Universitätsklinikum Freiburg, Medizinische Fakultät, Albert-Ludwigs-Universität Freiburg, Freiburg, Deutschland; ${ }^{20}$ Institut für Medizinische Epidemiologie, Biometrie und Informatik, Martin-LutherUniversität Halle-Wittenberg, Halle, Deutschland; ${ }^{21}$ Klinik für Allgemeine und Interventionelle Kardiologie, Universitäres Herz- und Gefäßzentrum Hamburg - Eppendorf, Hamburg, Deutschland; ${ }^{22}$ Epidemiologisches Studienzentrum, Universitätsklinikum Hamburg - Eppendorf, Hamburg, Deutschland; ${ }^{23}$ Institut für Medizinische Biometrie und Epidemiologie, Universitätsklinikum Hamburg Eppendorf, Hamburg, Deutschland; ${ }^{24}$ Abteilung Epidemiologie, Helmholtz-Zentrum für Infektionsforschung, Braunschweig, Deutschland; ${ }^{25}$ Institut für Epidemiologie, Christian-AlbrechtsUniversität Kiel, Kiel, Deutschland; ${ }^{26}$ Leipziger Forschungszentrum für Zivilisationserkrankungen (LIFE), Universität Leipzig, Leipzig, Deutschland; ${ }^{27}$ Institut für Medizinische Informatik, Statistik, und Epidemiologie (IMISE), Universität Leipzig, Leipzig, Deutschland; ${ }^{28}$ Abteilung Epidemiologie von Krebserkrankungen, DKFZ Heidelberg, Heidelberg, Deutschland; ${ }^{29}$ Translational Lung Research Center Heidelberg (TLRC), Deutsches Zentrum für Lungenforschung (DZL), Heidelberg, Deutschland; ${ }^{30}$ Institut für Epidemiologie und Sozialmedizin, Westfälische Wilhelms-Universität Münster, Münster, Deutschland; ${ }^{31}$ Institut für Community Medicine, Universitätsmedizin Greifswald, Greifswald, Deutschland; ${ }^{32}$ Institut für Community Medicine, Abt. Versorgungsepidemiologie und Community Health, Universitätsmedizin Greifswald, Greifswald, Deutschland; ${ }^{33}$ Institut für Epidemiologie und Präventivmedizin, Universität Regensburg, Regensburg, Deutschland; ${ }^{34}$ Abteilung Klinische Epidemiologie und Alternsforschung, Deutsches Krebsforschungszentrum (DKFZ), Heidelberg, Deutschland; ${ }^{35}$ Krebsregister Saarland, Ministerium für Soziales, Gesundheit, Frauen und Familie, Saarbrücken, Deutschland 